\title{
Quantum Groups at Odd Roots of Unity and Topological Invariants of 3-Manifolds
}

\author{
R.B. Zhang, A.L. Carey \\ Department of Pure Mathematics, University of Adelaide, Adelaide, Australia
}

Received: 16 October 1995/Accepted: 13 May 1996

\begin{abstract}
Topological invariants of three-manifolds are constructed using quantum groups associated with the $A, B, C$ and $D$ series of Lie algebras at odd roots of unity. These invariants are also explicitly computed for the lens spaces.
\end{abstract}

\section{Introduction}

Since the landmark discovery of Jones [1], there has been dramatic progress in the field of knot theory and the theory of 3-manifolds. A distinctive feature of the subject is its close connection with mathematical physics [2]. Indeed, many of the important discoveries are prompted by ideas and techniques from various branches of mathematical physics. For example, an intrinsically three dimensional construction of the Jones and related link polynomials was developed in terms of the quantum Chern-Simons theory [3]; new topological invariants of 3-manifolds were obtained using conformal field theory [4], and quantum groups $[5,6]$. It is our aim here to study 3-manifolds using quantum groups [7, 8].

We will apply the construction of Reshetikhin-Turaev $[5,6]$ to the quantum groups associated with the $A, B, C$ and $D$ series of Lie algebras at odd roots of unity to obtain the corresponding topological invariants of 3-manifolds. We will also compute these invariants for the lens spaces. Previously these quantum groups at even roots of unity were considered by Turaev and Wenzl [9, 6], who made essential use of a result of Kac-Peterson [10] on modular properties of characters of irreducible integrable representations of affine Lie algebras, which does not seem to apply to the odd roots of unity case in any obvious way. The HOMFLY and Kauffman ploynomials associated with these Lie algebras were also used in [20] to construct 3-manifold invariants, which were averages over all possible cablings [19].

The Reshetikhin-Turaev construction makes use of two fundamental theorems in 3-manifold theory, due to Lickorish [11] and Wallace, and Kirby [12] and Craggs respectively. The Lickorish-Wallace theorem states that each framed link in $S^{3}$ determines a closed, orientable 3-manifold, and every such 3-manifold is obtainable by surgery along a framed link in $S^{3}$. The disadvantage of this description 
of 3-manifolds is that different framed links may yield homeomorphic 3-manifolds upon surgery. This problem was resolved by Kirby [12] and Craggs, and Fenn and Rourke [13]. These authors proved that orientation preserving homeomorphism classes of closed, orientable 3-manifolds correspond bijectively to equivalence classes of framed links in $S^{3}$, where the equivalence relation is generated by the Kirby moves. The essential idea of [5] is to make appropriate combinations of isotopy invariants of a framed link embedded in $S^{3}$, such that they will be intact under the Kirby moves, and thus qualify as topological invariants of the 3-manifold obtained by surgery along this link.

It requires detailed knowledge of representations of quantum groups at roots of unity in order to explicitly carry out the Reshetikhin-Turaev construction. As the representation theory at roots of unity is not very well understood, this is by no means a straightforward exercise. In [5], the quantum group $U_{q}(s l(2))$ at even roots of unity was investigated, yielding a set of 3-manifold invariants which turned out to be equivalent to those arising from $s l(2)$ Chern-Simons theory. We should point out that it is the $\operatorname{sl}(2)$ invariants, which have been studied extensively, both within the framework of quantum groups and that of Chern-Simons theory [14-17], as well as using the cabling method $[19,20]$. In particular, these invariants were explicitly computed for the lens spaces [16]; the squares of their norms [6] were shown to be the same as the Turaev-Viro invariants [22]; their connections with the Casson invariants of homological spheres were also explored [23]. Also as we have already mentioned, Turaev and Wenzl [9] carried out the construction for quantum groups associated with the simple classical Lie algebras at even roots of unity. The resultant 3-manifold invariants are believed to be equivalent to those obtained from conformal field theory and Chern-Simons theory. In particular, the invariants arising from quantum $s l(n)$ at even roots of unity should be the same as those constructed in [24]. The Reshetikhin-Turaev construction was also extended to quantum supergroups [25], and the 3-manifold invariants associated with $U_{q}(g l(2 \mid 1))$ and $U_{q}(\operatorname{osp}(1 \mid 2))$ were obtained.

Our purpose is to study the case of the quantum groups associated with the simple classical Lie algebras at odd roots of unity. The organization of the paper is as follows. Section 2 summarizes the structural and representation theoretical properties of quantum groups, which will be necessary for the construction of 3-manifold invariants. Section 3 presents the construction of 3-manifold invariants, while Sect. 4 gives the result of the lens space computations.

\section{Quantum Groups}

2.1. At generic $q$. Let us begin by quickly reviewing the major properties of a quantum group $U_{q}(g)$. We will work on the complex field $C$. Let $g$ be any finite dimensional complex simple Lie algebra. Denote by $\Phi^{+}$the set of the positive roots of $g$ relative to a base (i.e., the set of the simple roots) $\Pi=\left\{\alpha_{1}, \ldots, \alpha_{l}\right\}$. Define $H^{*}=\bigoplus_{i=1}^{l} C \alpha_{i}, E=\bigoplus_{i=1}^{l} R \alpha_{i}$. Let $():, E \times E \rightarrow R$ be an inner product of $E$ such that the Cartan matrix $A$ of $g$ is given by

$$
A=\left(a_{i j}\right)_{i j=1}^{l}, a_{i j}=\frac{2\left(\alpha_{i}, \alpha_{j}\right)}{\left(\alpha_{i}, \alpha_{i}\right)} .
$$

Then Jimbo's version of the quantum group $U_{q}(g)$ is defined to be the unital associative algebra generated by $\left\{k_{i}, k_{i}^{-1}, e_{i}, f_{i} \mid i=1, \ldots, l\right\}$ with the following 
relations:

$$
\begin{aligned}
& k_{i} k_{j}=k_{j} k_{i}, \\
& k_{i} k_{i}^{-1}=1, \\
& k_{i} e_{j} k_{i}^{-1}=q^{\left(\alpha_{l}, \alpha_{j}\right)} e_{j}, \\
& k_{i} f_{j} k_{i}^{-1}=q^{-\left(\alpha_{l}, \alpha_{j}\right)} f_{j}, \\
& {\left[e_{i}, f_{j}\right] }=\delta_{i j} \frac{k_{i}-k_{i}^{-1}}{q-q^{-1}} \\
& \sum_{t=0}^{1-a_{l j}}(-1)^{t}\left[\begin{array}{lll}
1 & - & a_{i j} \\
& t &
\end{array}\right]_{q^{\left(\alpha_{i}, \alpha_{i}\right) / 2}}\left(e_{i}\right)^{t} e_{j}\left(e_{i}\right)^{1-a_{i j}-t}=0, \quad i \neq j, \\
& \sum_{t=0}^{1-a_{i j}}(-1)^{t}\left[\begin{array}{lll}
1 & - & a_{i j}
\end{array}\right]_{q^{\left(\alpha_{l}, \alpha_{l}\right) / 2}}\left(f_{i}\right)^{t} f_{j}\left(f_{i}\right)^{1-a_{l j}-t}=0, \quad i \neq j,
\end{aligned}
$$

where $\left[{ }_{t}^{s}\right]_{q}$ is the Gauss polynomial. (Note the slightly unusual normalization of $e_{i}$ and $f_{i}$ in the first equation of (2).)

Drinfeld's version of the quantum group on the other hand is defined to be an algebra over $C[[h]]$ completed with respect to the $h$-adic topology. It is generated by $\left\{h_{i}, e_{i}, f_{i} \mid i=1, \ldots, l\right\}$ subject to the same relations (1) and (2) with $k_{i}=q^{h_{i}}$, $q=\exp (h)$.

It is well known that (in either version) the quantum group $U_{q}(g)$ has the structure of a Hopf algebra. We will take the following co-multiplication:

$$
\begin{aligned}
\Delta\left(k_{i}^{ \pm 1}\right) & =k_{i}^{ \pm 1} \otimes k_{i}^{ \pm 1}, \\
\Delta\left(e_{i}\right) & =e_{i} \otimes k_{i}+1 \otimes e_{i}, \\
\Delta\left(f_{i}\right) & =f_{i} \otimes 1+k_{i}^{-1} \otimes f_{i},
\end{aligned}
$$

and co-unit:

$$
\begin{aligned}
\varepsilon\left(e_{i}\right) & =\varepsilon\left(f_{i}\right)=0, \\
\varepsilon\left(k_{i}^{ \pm 1}\right) & =\varepsilon(1)=1 .
\end{aligned}
$$

Then the antipode, which is an algebra anti-automorphism, is given by

$$
\begin{aligned}
S\left(k_{i}^{ \pm 1}\right) & =k_{i}^{\mp 1}, \\
S\left(e_{i}\right) & =-e_{i} k_{i}^{-1}, \\
S\left(f_{i}\right) & =-k_{i} f_{i} .
\end{aligned}
$$

Following Lusztig [26], we introduce a set of elements of $U_{q}(g)$

$$
\left\{e_{\alpha}^{(n)}, f_{\alpha}^{(n)} \mid \alpha \in \Phi^{+}, n \in Z_{+}\right\},
$$

which satisfy, for all $0 \leqq j \leqq \imath$,

$$
\begin{aligned}
k_{j} e_{\alpha}^{(n)} k_{j}^{-1} & =q^{n\left(\alpha, \alpha_{j}\right)} e_{\alpha}^{(n)}, \\
k_{j} f_{\alpha}^{(n)} k_{j}^{-1} & =q^{-n\left(\alpha, \alpha_{j}\right)} f_{\alpha}^{(n)} .
\end{aligned}
$$


The $e_{\alpha}^{(1)}$ and $f_{\alpha}^{(1)}$ have properties similar to that of the root spaces of the Lie algebra $g$. In particular, if $\alpha$ is simple, i.e., being equal to $\alpha_{i}$ for a given $i$, then $e_{\alpha}^{(1)}=e_{i}$, and $f_{\alpha}^{(1)}=f_{i}$.

At generic $q$, the Drinfeld quantum group admits a universal $R$-matrix, which can be expressed as

$$
R=q^{\sum_{i, j=1}^{l}\left(B^{-1}\right)_{l \jmath} h_{l} \otimes h_{\curlywedge}}\left(1 \otimes 1+\sum_{s} c_{(s)} E^{(s)} \otimes F^{(s)}\right),
$$

where $B=\frac{1}{2} \operatorname{diag}\left(\left(\alpha_{1}, \alpha_{1}\right), \ldots,\left(\alpha_{l}, \alpha_{l}\right)\right) A$. The $E^{(s)}$ and $F^{(s)}$ are ordered products of $e_{\alpha}^{(n)}$,s and $f_{\alpha}^{(n)}$, s respectively, while the $c_{(s)}$ are scalars, which are products of $q$-factorials and powers of $\left(q-q^{-1}\right)$.

The universal $R$-matrix satisfies the following defining relations:

$$
\begin{aligned}
R \Delta(a) & =\Delta^{\prime}(a) R, \quad \forall a \in U_{q}(g), \\
(\Delta \otimes \mathrm{id}) R & =R_{13} R_{23}, \\
(\mathrm{id} \otimes \Delta) R & =R_{13} R_{12},
\end{aligned}
$$

from which one can easily deduce that

$$
\begin{aligned}
& (S \otimes \mathrm{id}) R=\left(\mathrm{id} \otimes S^{-1}\right) R=R^{-1}, \\
& R_{12} R_{13} R_{23}=R_{23} R_{13} R_{12},
\end{aligned}
$$

where the second equation is the celebrated Yang-Baxter equation.

2.2. At roots of unity. When $q$ is a root of unity, Eqs. (1) and (2) still make sense within Jimbo's definition of a quantum group. However, our discussions about the universal $R$-matrix, etc. all become invalid. Many other things become rather subtle as well, thus requiring re-examination.

We assume that $q$ is a primitive $N^{\text {th }}$ root of 1 , with $N$ being an odd positive integer which is not smaller than the dual Coxeter number of the Lie algebra $g$. Let $\left\{\varepsilon_{i} \mid i=1,2, \ldots, r\right\}$ be a basis of an Euclidean space with inner product $\left(\varepsilon_{i}, \varepsilon_{j}\right)=\delta_{i j}$. Define

$$
X=\bigoplus_{i=1}^{r} Z \varepsilon_{i}, \quad X_{N}=X / N X
$$

For simplicity, we will work with the quantum groups associated with $A_{r-1}, B_{r}$, $C_{r}$ and $D_{r}$ only. The exceptional cases will be treated in a separate publication. In terms of the $\varepsilon_{i}$, the simple roots of these Lie algebras can be expressed in the standard way, which we spell out below.

$$
\begin{array}{cl}
\underline{A_{r-1}}: & \\
\underline{B_{r}}: & \alpha_{i}=\varepsilon_{i}-\varepsilon_{i+1}, \quad i=1, \ldots, r-1 ; \\
& \alpha_{i}=\varepsilon_{i}-\varepsilon_{i+1}, \quad i=1, \ldots, r-1, \quad \alpha_{r}=\varepsilon_{r} \\
\underline{C_{r}}: & \alpha_{i}=\varepsilon_{l}-\varepsilon_{i+1}, \quad i=1, \ldots r-1, \quad \alpha_{r}=2 \varepsilon_{r} ; \\
\underline{D_{r}:} & \alpha_{i}=\varepsilon_{i}-\varepsilon_{i+1}, \quad i=1, \ldots, r-1, \quad \alpha_{r}=\varepsilon_{r-1}+\varepsilon_{r+1} .
\end{array}
$$


Denote by $\imath$ the rank of the Lie algebra $g$. We define the quantum group $U_{q}^{(N)}(g)$ to be generated by $\left\{J_{a}, a=1, \ldots, r ; e_{i}, f_{i}, i=1, \ldots, l\right\}$, subject to the constraints (2) and the following relations:

$$
\begin{aligned}
J_{a} J_{b} & =J_{b} J_{a}, \\
J_{a} e_{i}\left(J_{a}\right)^{N-1} & =q^{\left(\alpha_{l}, \varepsilon_{a}\right)} e_{i}, \\
J_{a} f_{i}\left(J_{a}\right)^{N-1} & =q^{-\left(\alpha_{1}, \varepsilon_{a}\right)} f_{i}, \\
\left(J_{a}\right)^{N} & =1, \\
e_{\alpha_{l}}^{(N)}=f_{\alpha_{l}}^{(N)}=0, & \forall i=1,2, \ldots, l,
\end{aligned}
$$

where

$$
\begin{aligned}
& k_{i}=J_{i}\left(J_{i+1}\right)^{-1}, \quad i=1,2, \ldots, r-1 ; \\
& k_{r}= \begin{cases}J_{r}, & g=B_{r}, \\
\left(J_{r}\right)^{2}, & g=C_{r}, \\
J_{r-1} J_{r}, & g=D_{r} .\end{cases}
\end{aligned}
$$

Remarks. In the case of $A_{r-1}$, the quantum group we have just defined is that associated with $g l(r)$ instead of $s l(r)$. In choosing to work with $g l(r)$ we avoid complications related to the weight lattice of $\operatorname{sl}(r)$.

Our definition of $U_{q}^{(N)}(s o(n))$ automatically excludes the quantum analogues of spinorial type representations.

The algebra $U_{q}^{(N)}(g)$ still has the structure of a Hopf algebra, with the co-multiplication, co-unit and antipode for the $\left\{e_{i}, f_{i}\right\}$ generators as given in the generic $q$ case, and for the $J_{a}$ we have

$$
\begin{aligned}
\Delta\left(J_{a}^{ \pm 1}\right) & =J_{a}^{ \pm 1} \otimes J_{a}^{ \pm 1}, \\
\varepsilon\left(J_{a}^{ \pm 1}\right) & =1 ; \\
S\left(J_{a}^{ \pm 1}\right) & =J_{a}^{\mp 1} .
\end{aligned}
$$

It also admits a universal $R$-matrix, which reads

$$
R=\mathscr{K}\left(1 \otimes 1+\sum_{s} c_{(s)} E^{(s)} \otimes F^{(s)}\right),
$$

where $c_{(s)}, E^{(s)}$, and $F^{(s)}$ are the same as in the generic $q$ case, but the summation over $s$ is truncated by the extra relations

$$
e_{\alpha}^{(N)}=f_{\alpha}^{(N)}=0, \quad \forall \alpha \in \Phi^{+},
$$

which follow from (7) and the Lusztig automorphisms [27, 26]. Thus the sum involves only a finite number of terms. The factor $\mathscr{K}$ is defined by

$$
\mathscr{K}=\prod_{a=1}^{r}\left\{\sum_{\mu=0}^{N-1}\left(J_{a}\right)^{\mu} \otimes P_{a}[\mu]\right\},
$$


where the $P_{a}[\mu], a=1, \ldots, r, \mu=0, \ldots, N-1$, are idempotents defined by

$$
P_{a}[\mu]=\prod_{\substack{v=1 \\ v \neq \mu}}^{N-1} \frac{J_{a}-q^{v}}{q^{\mu}-q^{v}} .
$$

2.3. Representations. We now examine some facts about the representation theory of $U_{q}^{(N)}(g)$. Since ordered products of $e_{\alpha}^{(n)}, f_{\alpha}^{(n)}$ and powers of $J_{a}$ form a basis of $U_{q}^{(N)}(g)$, we can easily see that the quantum group is finite dimensional over $C$. It is a standard textbook result that every irreducible left module over an associative algebra is isomorphic to the quotient of the algebra itself by a maximal left ideal. Thus the irreducible representations of $U_{q}^{(N)}(g)$ are all finite dimensional. It can also be shown that every irreducible $U_{q}^{(N)}(g)$ module $V(\lambda)$ admits a unique (up to scalar multiples) highest and lowest weight vector, and is uniquely characterized by the highest weight $\lambda \in X_{N}$. Let $v_{+}$be a highest weight vector of $V(\lambda)$. Then by definition,

$$
\begin{aligned}
& J_{a} v_{+}=q^{\left(\lambda, \varepsilon_{a}\right)} v_{+}, \\
& e_{i} v_{+}=0,
\end{aligned}
$$

where we take an arbitrary representative of $\lambda$ in $X$ to evaluate $\left(\lambda, \varepsilon_{a}\right)$. Clearly $q^{\left(\lambda, \varepsilon_{a}\right)}$ is independent of the choice of the representative, thus is well defined on $X_{N}$. In a similar manner, we can also define a function $e: X_{N} \times X_{N} \rightarrow C$ by requiring that its pullback by the canonical projection $X \rightarrow X_{N}$ be given by $q^{(,)}: X \times X \rightarrow C$.

It is worth mentioning that in the cases of $B_{r}$ and $D_{r}$, no quantum analogues of the spinorial representations exist for $U_{q}^{(N)}(g)$. In the work of Turaev and Wenzl [9], such representations were also discarded.

Let

$$
\begin{aligned}
& \Lambda_{N}^{+}=\left\{\lambda \in X \mid 0<\frac{2(\lambda+\rho, \alpha)}{(\alpha, \alpha)}<N, \forall \alpha \in \Phi^{+}\right\} \\
& \overline{\Lambda_{N}^{+}}=\left\{\lambda \in X \mid 0 \leqq \frac{2(\lambda+\rho, \alpha)}{(\alpha, \alpha)} \leqq N, \forall \alpha \in \Phi^{+}\right\}
\end{aligned}
$$

Define $*: \Lambda_{N}^{+} \rightarrow \Lambda_{N}^{+}$by $\lambda \mapsto \lambda^{*}=-\tau(\lambda)$, where $\tau$ is the maximal element of the Weyl group of $g$. Then clearly $\Lambda_{N}^{+}$is stable under this map.

Note that the restriction of the canonical projection $X \rightarrow X / N X$ to $\Lambda_{N}^{+}$is one to one. We will continue to denote the image of $\Lambda_{N}^{+}$under this map by the same symbol. This deliberate abuse of notation will prove to be convenient.

Irreps of $U_{q}^{(N)}(g)$ with the highest weights belonging $\Lambda_{N}^{+}$are particularly interesting. They can be obtained from the $U_{q}(g)$ irreps with the same highest weights by specializing $q$ to the $N^{\text {th }}$ root of unity. Also, results of [20] and [28] state that

Theorem 1. The tensor product of any finite number of irreducible $U_{q}^{(N)}(g)$ modules $V\left(\lambda^{t}\right), \lambda^{t} \in \Lambda_{N}^{+}$, can be decomposed into

$$
\bigotimes_{t} V\left(\lambda^{t}\right)=\bigoplus_{\lambda \in \Lambda_{N}^{+}} V(\lambda)^{\oplus m(\lambda)} \oplus \mathscr{N},
$$


where $m(\lambda)$ is the multiplicity of $V(\lambda)$ appearing in the tensor product. The module $\mathscr{N}$ is a direct sum of indecomposable $U_{q}^{(N)}(g)$ modules, which has the property that for any module homomorphism $f: \mathscr{N} \rightarrow \mathscr{N}$, the quantum trace of $f$ vanishes identically, i.e.,

$$
\operatorname{tr}_{\mathscr{N}}\left(K_{2 \rho} f\right)=0 \text {. }
$$

Remarks A similar result also holds when $q$ is an even root of unity $[20,28]$.

In the case of the $B$ and $D$ series of quantum groups, the result of [28] involves quantum analogues of spinorial irreps. Since tensor products of tensorial irreps can not yield spinorial representations of any kind, the result still holds for $U_{q}^{(N)}(\operatorname{so}(n))$.

\section{Construction of Three-Manifold Invariants}

3.1. Technical results. In this subsection we present some technical results which will be of crucial importance for the remainder of the paper. Express the universal $R$ matrix of $U_{q}^{(N)}(g)$ as $R=\sum_{t} a_{t} \otimes b_{t}$, and define

$$
u=\sum_{t} S\left(b_{t}\right) a_{t}
$$

Then

$$
\begin{aligned}
u^{-1} & =\sum_{t} b_{t} S^{2}\left(a_{t}\right) \\
S^{2}(x) & =u x u^{-1}, \quad \forall x \in U_{q}^{(N)}(g) .
\end{aligned}
$$

Define

$$
K_{2 \rho}=\prod_{a=1}^{r}\left(J_{a}\right)^{\left(2 \rho, \varepsilon_{a}\right)}
$$

where $2 \rho=\sum_{\alpha \in \Phi^{+}} \alpha$ is the sum of the positive roots of $g$. Set

$$
v=u K_{2 \rho}^{-1} .
$$

Then $v$ is central, and satisfies

$$
\Delta(v)=(v \otimes v)\left(R^{T} R\right)^{-1} .
$$

Acting on an irreducible $U_{q}^{(N)}(g)$ module $V(\lambda)$ with highest weight $\lambda, v$ takes the eigenvalue

$$
\chi_{\lambda}(v)=q^{-(\lambda+2 \rho, \lambda)}
$$

where $q^{-(\lambda+2 \rho, \lambda)}=e(\lambda+2 \rho,-\lambda)$.

Let $V$ be a finite dimensional $U_{q}^{(N)}(g)$ module. We denote the corresponding representation by $\pi$. Define

$$
C_{V}=\operatorname{tr}_{V}\left[(\pi \otimes \mathrm{id})\left(K_{2 \rho}^{-1} \otimes 1\right) R^{T} R\right],
$$

where $\operatorname{tr}_{V}$ represents the trace taken over $V$. Then $C_{V}$ belongs to the central algebra of $U_{q}^{(N)}(g)$ [30]. 
If $V=V(\lambda)$ is an irreducible module with highest weight $\lambda \in \Lambda_{N}^{+}$, we denote the associated central element $C_{V}$ by $C_{\lambda}$. Acting on an irreducible $U_{q}^{(N)}(g)$ module $V(\mu)$ with highest weight $\mu \in \Lambda_{N}^{+}, C_{\lambda}$ takes the eigenvalue

$$
\chi_{\mu}\left(C_{\lambda}\right)=\operatorname{tr}_{V(\lambda)}\left(q^{2 h_{\mu+\rho}}\right)=S_{\lambda \mu} / Q(\mu)
$$

with

$$
\begin{aligned}
Q(\mu) & =\sum_{\sigma \in \mathscr{W}} \operatorname{det} \sigma q^{2(\sigma(\rho), \mu+\rho)}, \\
S_{\lambda \mu} & =\sum_{\sigma \in \mathscr{W}} \operatorname{det} \sigma q^{2(\sigma(\lambda+\rho), \mu+\rho)},
\end{aligned}
$$

where $\mathscr{W}$ represents the Weyl group of the Lie algebra $g$. The eigenvalue of $C_{\lambda}$ in the trivial irrep reads $Q(\lambda) / Q(0)$, which coincides with the $q$-dimension of $V(\lambda)$. We will denote it by $D_{q}(V(\lambda))$.

Some properties of the matrix $\left(S_{\lambda \mu}\right)$ are worth observing:

$$
\begin{aligned}
S_{\lambda \mu} & =S_{\mu \lambda}, \\
S_{\sigma(\lambda+\rho)-\rho, \mu} & =\operatorname{det} \sigma S_{\lambda \mu} .
\end{aligned}
$$

It immediately follows the second identity that $S_{\lambda_{v}}=0$ if $\lambda \in \overline{\Lambda_{N}^{+}}-\Lambda_{N}^{+}$.

We wish to find a set of complex numbers $d_{\lambda}, \lambda \in \Lambda_{N}^{+}$, which render the central element of $U_{q}^{(N)}(g)$ defined by

$$
\delta=v-\sum_{\lambda \in \Lambda_{N}^{+}} d_{\lambda} \chi_{\lambda}\left(v^{-1}\right) C_{\lambda},
$$

vanishing on all irreducible $U_{q}^{(N)}(g)$ modules with highest weights contained in $\Lambda_{N}^{+}$.

Lemma 1. i) There exists at least one set of $d_{\lambda}, \lambda \in \Lambda_{N}^{+}$, such that

$$
d_{\lambda}=d_{\lambda^{*}}, \quad \forall \lambda \in \Lambda_{N}^{+},
$$

and $\delta$ takes zero eigenvalue in all irreducible $U_{q}^{(N)}(g)$ modules with highest weights belonging to $\Lambda_{N}^{+}$.

ii) One such set is given by

$$
\begin{aligned}
& d_{\lambda}=\Omega Q(\lambda), \quad \lambda \in \Lambda_{N}^{+}, \\
& \Omega=(-1)^{\left|\Phi^{+}\right|} \frac{q^{\left(1+\frac{N+1}{2}\right)(2 \rho, \rho)}}{\left(G_{1}(q)\right)^{r}} .
\end{aligned}
$$

Proof. The vanishing of the central element $\delta$ in an irreducible $U_{q}^{(N)}(g)$ module $V(\mu), \mu \in \Lambda_{N}^{+}$, is equivalent to the following equation:

$$
Q(\mu) q^{-(\mu+2 \rho, \mu)}=\sum_{\lambda \in \Lambda_{N}^{+}} d_{\lambda} q^{(\lambda+2 \rho, \lambda)} S_{\lambda \mu}
$$

Therefore, for the purpose of proving the lemma, it suffices to construct a set of $d_{\lambda}$, $\lambda \in \Lambda_{N}^{+}$, which satisfy (14) for all $\mu \in \Lambda_{N}^{+}$. 
Instead of constructing the $d_{\lambda}$ 's directly, we solve the following auxiliary equation for the unknowns $x_{\lambda}, \lambda \in X_{N}$ :

$$
Q(\mu) q^{-(\mu+2 \rho, \mu)}=\sum_{\lambda \in X_{N}} x_{\lambda} q^{(\lambda+2 \rho, \hat{\lambda})} S_{\lambda \mu}
$$

Note the difference between this equation and (14): the sum on the right-hand side is now over $X_{N}$. Let us try the following ansatz:

$$
x_{\lambda}=c q^{-2(\lambda+\rho, \rho)}
$$

where $c$ is a $\lambda$ independent complex number. Inserting it into the right-hand side of (15), we obtain

$$
\sum_{\lambda \in X_{N}} x_{\lambda} q^{(\lambda+2 \rho, \lambda)} S_{\lambda \mu}=c \sum_{\sigma \in \mathscr{W}} \operatorname{det} \sigma q^{2(\mu+\rho, \sigma(\rho))} \sum_{\lambda \in X_{N}} q^{(\lambda, \lambda)+2(\lambda, \sigma(\mu+\rho))-2(\rho, \rho)} .
$$

Recall that $X_{N}=X / N X$, where $X$ is the root lattice in the case of $B_{r}, C_{r}$ and $D_{r}$ series of Lie algebras, and in the case of the $A$ series, is an integral lattice in the dual of the Cartan subalgebra of $g l(r)$ (instead of $s l(r)$ ). We can easily see that the variable of the summation on the right-hand side can be translated by any element of $X_{N}$ without affecting the final result. (However, we must note that translating the variable by things like $\varepsilon_{i} / 2+N X$ is not allowed.) Shifting the summation variable by $\frac{N+1}{2} \cdot 2 \sigma(\mu+\rho)$, we arrive at

$$
\sum_{\lambda \in X_{N}} x_{\lambda} q^{(\lambda+2 \rho, \lambda)} S_{\lambda \mu}=c q^{-\left(1+\frac{N+1}{2}\right)(2 \rho, \rho)} \sum_{\lambda \in X_{N}} q^{(\lambda, \lambda)} \times q^{-(\mu+2 \rho, \mu)} \sum_{\sigma \in \mathscr{W}} \operatorname{det} \sigma q^{2(\mu+\rho, \sigma(\rho))},
$$

which uniquely determines

$$
c=q^{\left(1+\frac{N+1}{2}\right)(2 \rho, \rho)} / \sum_{\lambda \in X_{N}} q^{(\lambda, \lambda)} .
$$

Set

$$
G_{k}(q)=\sum_{j=0}^{N-1} q^{k j^{2}}
$$

For all the quantum groups $U_{q}^{(N)}(g)$ which we are considering,

$$
\sum_{\lambda \in X_{N}} q^{(\lambda, \hat{\lambda})}=\left(G_{1}(q)\right)^{r}
$$

where the result holds for $U_{q}^{(N)}(s o(n))$ as well because the spinorial representations are excluded automatically. Therefore

$$
x_{\lambda}=\frac{q^{\left(1+\frac{N+1}{2}\right)(2 \rho, \rho)-2(\lambda+\rho, \rho)}}{\left(G_{1}(q)\right)^{r}}, \quad \lambda \in X_{N},
$$

is a solution of (15). 
To construct the $d_{\lambda}$ from the $x_{v}$, we consider the affine Weyl group $\mathscr{W}_{N}$ generated by

$$
\begin{aligned}
S_{\alpha, k N}: X \otimes_{Z} R & \rightarrow X \otimes_{Z} R, \quad \alpha \in \Phi^{+}, k \in Z, \\
\mu & \mapsto \sigma_{\alpha}(\mu+\rho)-\rho+k N \alpha,
\end{aligned}
$$

where $\sigma_{\alpha} \in \mathscr{W}$ is the reflection associated with the root $\alpha$ of $g$. It is a standard result that $\mathscr{W}_{N}$ acts on the chambers, i.e., the open connected components of

$$
X \otimes_{Z} R-\bigcup_{\alpha \in \Phi^{+}} \bigcup_{n \in Z}\left\{\begin{array}{l|l}
\mu \in X \otimes_{Z} R & \frac{2(\mu+\rho, \alpha)}{(\alpha, \alpha)}=n N
\end{array}\right\},
$$

simply transitively, with a fundamental domain

$$
\left\{\mu \in X \otimes_{Z} R \mid 0 \leqq \frac{2(\mu+\rho, \alpha)}{(\alpha, \alpha)} \leqq N, \quad \forall \alpha \in \Phi^{+}\right\} .
$$

We define the action of $\mathscr{W}_{N}$ on $X_{N}$ by

$$
S_{\alpha, k N}(\mu+N X)=S_{\alpha, k N}(\mu)+N X,
$$

which clearly coincides with the action of the ordinary Weyl group $\mathscr{W}$ on $X_{N}$ defined by

$$
\sigma(\lambda+\rho+N X)=\sigma(\lambda+\rho)+N X, \quad \forall \sigma \in \mathscr{W}, \lambda \in X
$$

From the two properties of $\mathscr{W}_{N}$ on $X \otimes_{Z} R$ we can deduce that the image of $\overline{\Lambda_{N}^{+}}$ under the canonical mapping $X \rightarrow X_{N}$ furnishes a fundamental domain for the action of $\mathscr{W}$. For any $\lambda, \mu \in \Lambda_{N}^{+} \subset X_{N}$ and $\sigma, \omega \in \mathscr{W}, \sigma(\lambda+\rho)-\rho, \omega(\mu+\rho)-\rho \in X_{N}$ have the following important property:

$$
\sigma(\lambda+\rho)-\rho=\omega(\mu+\rho)-\rho \quad \text { iff } \sigma=\omega, \quad \lambda=\mu .
$$

The properties of $\Lambda_{N}^{+}$under the action of the Weyl group and the fact that $S_{\lambda v}=0$ if $\lambda \in \overline{\Lambda_{N}^{+}}-\Lambda_{N}^{+}$allow us to re-write

$$
\sum_{\nu \in X_{N}} x_{\nu} q^{(\lambda+2 \rho, v)} S_{\nu \mu}=\sum_{\lambda \in \Lambda_{N}^{+}} \sum_{\sigma \in \mathscr{W}} \operatorname{det} \sigma x_{\sigma(\lambda+\rho)-\rho} q^{(\lambda+2 \rho, \lambda)} S_{\lambda \mu}
$$

which immediately yields

$$
d_{\lambda}=\sum_{\sigma \in \mathscr{W}} \operatorname{det} \sigma x_{\sigma(\lambda+\rho)-\rho}, \quad \lambda \in \Lambda_{N}^{+} .
$$

Now Eq. (13) follows directly, and it is also clear that $d_{\lambda}=d_{\lambda^{*}}$.

Several features of the $d_{\lambda}$ are worth observing. Recall that the $q$-dimension of the irreducible $U_{q}^{(N)}(g)$ module $V(\lambda)$ with highest weight $\lambda \in \Lambda_{N}^{+}$is given by

$$
D_{q}(V(\lambda))=Q(\lambda) / Q(0) .
$$

Clearly $d_{\lambda}$ is proportional to the $q$-dimension,

$$
d_{\lambda}=d_{0} D_{q}(V(\lambda)), \quad d_{0}=\Omega Q(0), \forall \lambda \in \Lambda_{N}^{+} .
$$

This phenomenon also appeared in the even $N$ case $[9,20]$. 
Retrospectively, if $d_{0}$ were unknown, and we merely postulate that $d_{\lambda}$ is proportional the $q$-dimension, then the $\mu=0$ case of (14) requires that the proportionality constant $d_{0}$ obey the following relation:

$$
d_{0}^{-1}=\sum_{\lambda \in \Lambda_{N}^{+}} q^{(\lambda+2 \rho, \lambda)}\left(D_{q}(\lambda)\right)^{2} .
$$

A direct evaluation indeed yields $\Omega Q(0)$ for the right-hand side, providing a consistency check for our result.

From now on we will take $\delta$ as being defined by (11) with the $d_{\lambda}$ coefficients given by (13). We also define the set

$$
\mathscr{V}\left(\Lambda_{N}^{+}\right)=\left\{V(\lambda) \mid \lambda \in \Lambda_{N}^{+}\right\}
$$

of all the irreducible $U_{q}^{(N)}(g)$ modules with highest weights belonging to $\Lambda_{N}^{+}$. An important property of the central element $\delta$ with the $d_{\lambda}$ 's given by (13) is

Lemma 2. Let $Y$ be a $U_{q}^{(N)}(g)$ module which is the tensor product of a finite number of elements (not necessarily distinct) of $\mathscr{V}\left(\Lambda_{N}^{+}\right)$. For any module homomorphism $f: Y \rightarrow Y$,

$$
\operatorname{tr}_{Y}\left(K_{2 \rho} \delta f\right)=0,
$$

where $\operatorname{tr}_{Y}$ represent the trace taken over $Y$.

Proof. It immediately follows from the definition of $\delta$ and Theorem 1.

Let us define

$$
z=\sum_{\lambda \in \Lambda_{N}^{+}} d_{\lambda} q^{-(\lambda+2 \rho, \lambda)} D_{q}(\lambda)
$$

where $D_{q}(\lambda)$ denotes the quantum dimension of the irreducible $U_{q}^{(N)}(g)$ module $V(\lambda) \in \mathscr{V}\left(\Lambda_{N}^{+}\right)$. Then we have

$$
z=(-1)^{\left|\Phi^{+}\right|}\left(\frac{G_{N-1}(q)}{\sqrt{N}}\right)^{2 r} q^{\left(1+\frac{N+1}{2}\right)(2 \rho, 2 \rho)}, \quad \frac{G_{N-1}(q)}{\sqrt{N}}=-i(-1)^{(N+1) / 2} .
$$

By using the "strange formula," $z$ can be cast into the form $\pm \exp ^{\left(-i 2 \eta \pi \frac{(2 N-\check{h}) \operatorname{dim} g^{\prime}}{2 N}\right)}$, where $g^{\prime}=[g, g]$, and $\breve{h}$ is the dual Coxeter number of this Lie algebra. The $\eta$ is 2 for $\operatorname{sp}(2 r)$ and 1 for all other cases. Furthermore, if we set $\mathscr{D}^{2}=\sum_{\lambda \in \Lambda_{N}^{+}}\left(D_{q}(\lambda)\right)^{2}$, then direct calculations give $z=d_{0}^{2} \mathscr{D}^{2}$.

3.2. Three-manifold invariants. In the construction of 3-manifold invariants using quantum groups, the Reshetikhin-Turaev functor from the category of coloured ribbon graphs to the category of finite dimensional representations of quantum groups plays the central role. For detailed discussions of coloured ribbon graphs and the functor, we refer to [6]. We will not need to consider the full category in this paper, rather, the subcategory consisting only of the coloured ribbon graphs without coupons will suffice for us. We will adopt the same convention as that of [25], which differs from that of [6].

As in the last section, we denote by $V(\lambda)$ the irreducible $U_{q}^{(N)}(g)$ module with highest weight $\lambda$, and $\mathscr{V}\left(\Lambda_{N}^{+}\right)$the set of the irreducible $U_{q}^{(N)}(g)$ modules with highest weights belonging to $\Lambda_{N}^{+}$. As we have already pointed out, if $V(\mu) \in \mathscr{V}\left(\Lambda_{N}^{+}\right)$, 
then its dual module $V(\mu)^{*}=V\left(\mu^{*}\right)$ also belongs to $\mathscr{V}\left(\Lambda_{N}^{+}\right)$. Furthermore, no two elements of $\mathscr{V}\left(\Lambda_{N}^{+}\right)$are isomorphic if their highest weights are distinct. We will colour ribbon graphs with irreps in $\mathscr{V}\left(\Lambda_{N}^{+}\right)$.

Consider as examples the ribbon $(k, k)$ graphs depicted in Fig. 1.

We colour the ribbons of both Fig. $1 \mathrm{a}$ and $1 \mathrm{~b}$ by $\left\{V\left(\lambda_{1}\right), V\left(\lambda_{2}\right), \ldots, V\left(\lambda_{k}\right)\right\}$, while colour the annulus of Fig. 1a by the irreducible module $V(\mu)$. We denote the resultant coloured ribbon graphs by $\phi_{\mu}^{(k)}$ and $\zeta^{(k)}$ respectively. It is straightforward to obtain,

$$
\begin{aligned}
& F\left(\phi_{\mu}^{(k)}\right)=\chi_{j}\left(v^{-1}\right) \Delta^{(k-1)}\left(C_{\mu}\right), \\
& F\left(\zeta^{(k)}\right)=\Delta^{(k-1)}(v),
\end{aligned}
$$

which map $V\left(\lambda_{1}\right) \otimes V\left(\lambda_{2}\right) \otimes \cdots \otimes V\left(\lambda_{k}\right)$ to itself.

To construct topological invariants of a closed, oriented 3-manifold $M_{L}$, we first give it a surgery description, namely, representing it by surgery along a framed link $L$ embedded in $S^{3}$. The fundamental theorem of Lickorish-Wallace asserts that every closed, oriented 3-manifold can be described this way. Then we make appropriately weighted sums of the framed link invariants of $L$ associated with different representations in such a way that the final combinations are invariant under the Kirby moves. It follows from Kirby's theorem that these combinations are homeomorphism invariants of the 3-manifold.

Assume that the framed link $L$ (in blackboard framing) consists of $m$ components $L_{i}, i=1, \ldots, m$. It gives rise to a unique ribbon graph by extending each component $L_{i}$ to an annulus, which has $L_{i}$ itself and an $L_{i}^{\prime}$ as its edges, where $L_{i}^{\prime}$ is a parallel copy of $L_{i}$ such that the linking number between the two is equal to the framing number of the latter. We denote this ribbon graph by $\Gamma(L)$.

We colour $\Gamma(L)$ by associating with each component $L_{i}$ with a $V\left(\lambda_{i}\right) \in \mathscr{V}\left(\Lambda_{N}^{+}\right)$. Set $c=\left\{\lambda^{(1)}, \lambda^{(2)}, \ldots, \lambda^{(m)}\right\}$, where some $\lambda^{(j)}$ 's may be equal, and denote by $\mathscr{C}(L)$ the set of all the distinct $c$ 's. The ribbon graph coloured by modules associated with $c$ will be denoted by $\Gamma_{c}(L)$. The Reshetikhin-Turaev functor applied to $\Gamma_{c}(L)$ yields $F\left(\Gamma_{c}\right)$, which is a homomorphism of the trivial $U_{q}^{(N)}(g)$ module to itself, thus is a complex number.

We define

$$
\Sigma(L)=\sum_{c \in \mathscr{C}(L)} \prod_{l=1}^{m} d_{\lambda^{(l)}} F\left(\Gamma_{c}(L)\right),
$$
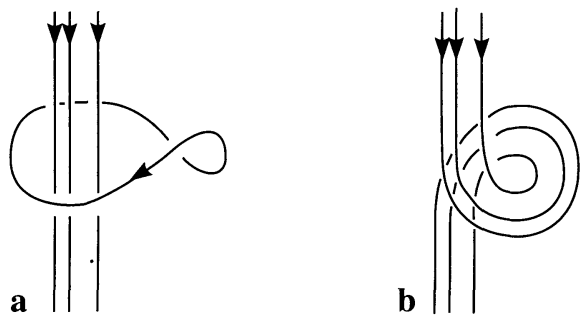

Fig. 1. 
where the $d_{\lambda}$ are the set of constants given in (13). $\Sigma(L)$ has the following important properties:

Proposition 1. $\Sigma(L)$ is independent of the orientation chosen for $L$, and also invariant under the positive Kirby moves depicted in Fig. $2 a$ and Fig. $2 b$.

Proof. The first statement of the theorem follows from the fact that $d_{\lambda}=d_{\lambda^{*}}$, $\forall \lambda \in \Lambda_{N}^{+}$, while the second statement is a direct consequence of the lemmas of the last section and Eq. (18).

On the other hand, $\Sigma$ is not invariant under the Kirby $(-)$ moves given in Fig. $2 \mathrm{c}$ and $2 \mathrm{~d}$. In particular, if $L^{\prime}$ is the framed link obtained by applying once the special Kirby (-) move Fig. 2c, namely, adding a framing -1 unknot, to the framed link $L$, then

$$
\Sigma\left(L^{\prime}\right)=z \Sigma(L)
$$

that is, under a special Kirby (-) move, $\Sigma$ is scaled by $z$. Since $|z|=1$, the square of the norm of $\Sigma$ remains intact under both the Kirby $(+)$ moves and the special Kirby $(-)$ move. In view of the fact that these moves together generate the entire Kirby calculus, we conclude that

Proposition 2. The following quantity

$$
\nabla\left(M_{L}\right)=|\Sigma(L)|^{2}
$$

is a topological invariant of $M_{L}$.

However, $\nabla\left(M_{L}\right)$ is not the Reshetikhin-Turaev invariant, which we aim to obtain. To achieve that, further work is required. We consider the matrix $A_{L}=$ $\left(a_{i j}\right)_{m \times m}$ which is the intersection form on the second homology group $H_{2}\left(W_{L}, Z\right)$ of

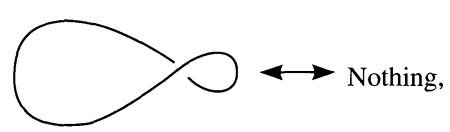

a

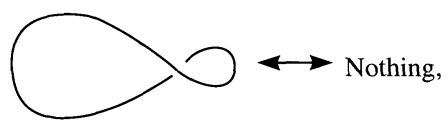

c

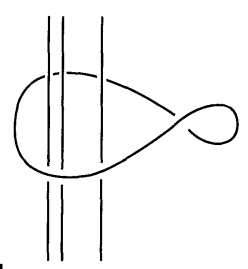

b

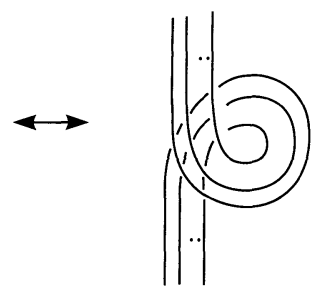

d
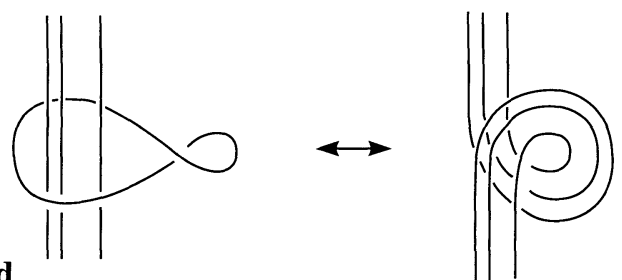

Fig. 2. 
$W_{L}$, where $W_{L}$ is the 4-manifold bounded by $M_{L}$. A more down to earth interpretation of $A_{L}$ is the linking matrix of $L$ defined in the following way: $a_{i l}$ is equal to the framing number of the $i^{\text {th }}$ component of $L$, and $a_{i j}, i \neq j$ is the linking number between the $i^{\text {th }}$ and $j^{\text {th }}$ components of $L$. Let $\sigma\left(A_{L}\right)$ be the number of nonpositive eigenvalues of $A_{L}$, then it is clear that $\sigma\left(A_{L}\right)=\sigma\left(A_{L^{\prime}}\right)-1$, while the positive Kirby moves leave $\sigma\left(A_{L}\right)$ unchanged.

Therefore $z^{-\sigma\left(A_{L}\right)} \Sigma(L)$ is invariant under the positive Kirby moves and the special negative Kirby move, thus

Theorem 2. We have the following topological invariant of the 3-manifold $M_{L}$ :

$$
\mathscr{F}\left(M_{L}\right)=z^{-\sigma\left(A_{L}\right)} \Sigma(L)
$$

Remarks. There exist several versions of the Reshetikhin-Turaev invariants in the literature, e.g., those given in [5,9 and 6]. Although the kernel of all those invariants is $\Sigma(L)$, they differ nontrivially (i.e., $L$ dependently) in the factors multiplying $\Sigma(L)$. Our definition follows that of [5],

In the special case of $g l(2),(21)$ is closely related to the topological invariant of [31].

\section{Computation of Quantum Invariants}

We compute the quantum invariant $\mathscr{F}$ for the Lens spaces $L(m, n)$, where $m, n \in Z$ are co-prime. Observe that different pairs of co-prime integers $(m, n)$ and $\left(m^{\prime}, n^{\prime}\right)$ can lead to homeomorphic manifolds. To avoid (some) repetitions, we assume that $0<n<m$. This exhausts all the possible lens spaces apart from $S^{3}$ and $S^{2} \times S^{1}$, which can be treated separately. For any such pair $(m, n)$, there always exists a unique set of integers $\left\{a_{1}, \ldots, a_{s}\right\}$ for some $s$ with $2 \leqq a_{i} \in Z$, such that the ratio $m / n$ can be expressed as a continued fraction

$$
\frac{m}{n}=a_{1}-\frac{1}{a_{2}-\frac{1}{a_{3}-\frac{1}{-\frac{1}{a_{s-1}-\frac{1}{a_{s}}}}}} .
$$

Consider the framed link given by Fig. 3 .

The integers $a_{1}, a_{2}, \ldots, a_{s}$ are now the framing numbers of the respective components of the link. Surgery along this framed link yields $L(m, n)$.

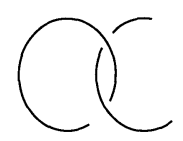

$\mathrm{a}_{\mathrm{s}}$

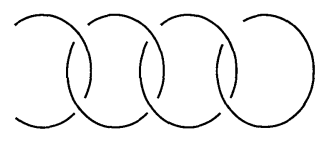

$\mathrm{a}_{2} \quad \mathrm{a}_{1}$

Fig. 3. 


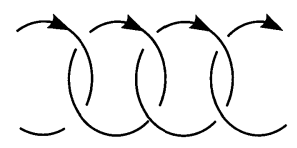

$\mathrm{a}_{\mathrm{k}}$

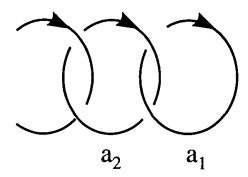

Fig. 4.

In order to compute the topological invariant $\mathscr{F}(L(m, n))$, we need some preparations. For each $\lambda \in X_{N}$, we define

$$
\varpi_{\lambda}= \begin{cases}d_{\lambda} / Q(\lambda), & \lambda \in \Lambda_{N}^{+}, \\ 0, & \lambda \notin \Lambda_{N}^{+}\end{cases}
$$

Then from the discussions on the properties of $X_{N}$ and $\Lambda_{N}^{+}$under the Weyl group $\mathscr{W}$ we can easily deduce that

$$
\sum_{\sigma \in \mathscr{W}} \varpi_{\sigma(\lambda+\rho)-\rho}= \begin{cases}\Omega, & \frac{2(\lambda+\rho, \alpha)}{(\alpha, \alpha)} \neq 0(\bmod N), \quad \forall \alpha \in \Phi^{+} \\ 0, & \text { otherwise }\end{cases}
$$

Let us consider the coloured ribbon graph given in Fig. 4.

We denote the graph by $\Gamma\left(a_{1}, \ldots, a_{k} ; \mu_{1}, \ldots, \mu_{k}\right)$. The $i^{\text {th }}$ annulus from the right has $2 a_{i}$ positive twists, and is coloured by the irreducible $U_{q}^{(N)}(g)$ module $V\left(\mu_{i}\right)$, with $\mu_{i} \in \Lambda_{N}^{+}$. The ribbon is coloured by $V(\lambda), \lambda \in \Lambda_{N}^{+}$.

Define

$$
\begin{aligned}
H_{\lambda}^{(k)}\left(a_{1}, \ldots, a_{k}\right) & =\sum_{\mu_{1}, \mu_{k} \in \Lambda_{N}^{+}} \prod_{i=1}^{k} d_{\mu_{l}} F\left(\Gamma\left(a_{k}, \ldots, a_{k} ; \mu_{1}, \ldots, \mu_{k}\right)\right), \\
h_{\lambda}^{(k)}\left(a_{1}, \ldots, a_{k}\right) & =Q(\lambda) H_{\lambda}^{(k)}\left(a_{1}, \ldots, a_{k}\right) .
\end{aligned}
$$

It is easy to see that

$$
H_{\lambda}^{(1)}\left(a_{1}\right)=\frac{\Omega}{Q(\lambda)} \sum_{\mu_{1} \in X_{N}} Q\left(\mu_{1}\right) q^{a_{1}\left(\mu_{1}+2 \rho, \mu_{1}\right)+2\left(\mu_{1}+\rho, \lambda+\rho\right)} .
$$

Note that the sum on the right-hand side is over $X_{N}$ instead of $\Lambda_{N}^{+}$. It is also important to observe that although $H_{\lambda}^{(1)}\left(a_{1}\right)$ is only defined for $\lambda \in \Lambda_{N}^{+}, h_{\lambda}^{(1)}\left(a_{1}\right)$ makes perfect sense for all $\lambda \in X_{N}$. Furthermore,

$$
h_{\lambda}^{(1)}\left(a_{1}\right)=\operatorname{det} \sigma h_{\sigma(\lambda+\rho)-\rho}^{(1)}\left(a_{1}\right), \quad \forall \sigma \in \mathscr{W} .
$$

This in particular implies that if there is an $\alpha \in \Phi^{+}$such that $\frac{2(\lambda+\rho, \alpha)}{(\alpha, \alpha)} \equiv 0(\bmod N)$, then $h_{\lambda}^{(1)}\left(a_{1}\right)$ vanishes identically.

$H_{\lambda}^{(2)}\left(a_{1}, a_{2}\right)$ can be expressed as

$$
H_{\lambda}^{(2)}\left(a_{1}, a_{2}\right)=\frac{1}{Q(\lambda)} \sum_{\mu_{2} \in \Lambda_{N}^{+}} \varpi_{\mu_{2}} \sum_{\sigma \in \mathscr{W}} \operatorname{det} \sigma q^{a_{2}\left(\mu_{2}+2 \rho, \mu_{2}\right)} q^{2\left(\sigma\left(\mu_{2}+\rho\right), \hat{\lambda}+\rho\right)} h_{\mu_{2}}^{(1)}\left(a_{1}\right) .
$$


In the above equation, the sum over $\mu_{2}$ can be extended to the entire set $X_{N}$ without affecting $H_{\lambda}^{(2)}\left(a_{1}, a_{2}\right)$ in any way. By using Eq. (22) we can cast it into the form

$$
H_{\lambda}^{(2)}\left(a_{1}, a_{2}\right)=\frac{\Omega}{Q(\lambda)} \sum_{\mu \in X_{N}} q^{a_{2}(\mu+2 \rho, \mu)+2(\mu+\rho, \lambda+\rho)} h_{\mu}^{(1)}\left(a_{1}\right) .
$$

Note again that $h_{\lambda}^{(2)}\left(a_{1}, a_{2}\right)$ has similar properties as $h_{\lambda}^{(1)}\left(a_{1}\right)$, namely, it is well defined for all $\lambda \in X_{N}$, and

$$
h_{\lambda}^{(2)}\left(a_{1}, a_{2}\right)=\operatorname{det} \sigma h_{\sigma(\lambda+\rho)-\rho}^{(2)}\left(a_{1}, a_{2}\right), \quad \forall \sigma \in \mathscr{W} .
$$

By repeated applications of (22), we can easily establish the recursive formula

$$
h_{\lambda}^{(k)}\left(a_{1}, \ldots, a_{k}\right)=\Omega \sum_{\mu \in X_{N}} q^{a_{k}(\mu+2 \rho, \mu)+2(\mu+\rho, \lambda+\rho)} h_{\mu}^{(k-1)}\left(a_{1}, \ldots, a_{k-1}\right),
$$

which immediately leads to

$$
H_{\lambda}^{(k)}\left(a_{1}, \ldots, a_{k}\right)=\frac{\Omega^{k}}{Q(\lambda)} \sum_{\mu_{1}, \mu_{k} \in X_{N}} Q\left(\mu_{1}\right) q^{\sum_{l=1}^{k}\left[a_{i}\left(\mu_{l}+2 \rho, \mu_{l}\right)+2\left(\mu_{l}+\rho, \mu_{l+1}+\rho\right)\right]},
$$

where $\mu_{k+1}=\lambda \in \Lambda_{N}^{+}$.

Setting $\lambda=0$ in $H_{\lambda}^{(s)}\left(a_{1}, \ldots, a_{s}\right)$, we arrive at

$$
\Sigma(L(m, n))=H_{0}^{(s)}\left(a_{1}, \ldots, a_{s}\right) .
$$

The linking matrix for the framed link of Fig. 3 is given by

$$
\left(\begin{array}{lllllll}
a_{1} & 1 & 0 & 0 & \cdots & 0 & 0 \\
1 & a_{2} & 1 & 0 & \cdots & 0 & 0 \\
0 & 1 & a_{3} & 1 & \cdots & 0 & 0 \\
0 & 0 & 1 & a_{4} & \cdots & 0 & 0 \\
\cdots & \cdots & \cdots & \cdots & \cdots & \cdots & \cdots \\
0 & 0 & 0 & 0 & \cdots & a_{s-1} & 1 \\
0 & 0 & 0 & 0 & \cdots & 1 & a_{s}
\end{array}\right)
$$

where $2 \leqq a_{i} \in Z, \forall i$. It is quite obvious that the matrix is positive definite. Thus

$$
\mathscr{F}(L(m, n))=H_{0}^{(s)}\left(a_{1}, \ldots, a_{s}\right) .
$$

The two degenerate cases, $S^{3}$ and $S^{2} \times S^{1}$, are easy to handle. We have

$$
\begin{aligned}
\mathscr{F}\left(S^{3}\right) & =1, \\
\mathscr{F}\left(S^{2} \times S^{1}\right) & =z^{-1} \sum_{\lambda \in \Lambda_{N}^{+}} d_{\lambda} Q(\lambda) / Q(0) .
\end{aligned}
$$

To evaluate $\mathscr{F}\left(S^{2} \times S^{1}\right)$ more explicitly, we apply (22) to obtain

$$
z^{-1} \sum_{\lambda \in \Lambda_{N}^{+}} d_{\lambda} Q(\lambda) / Q(0)=z^{-1} \Omega(-1)^{\left|\Phi^{+}\right|} \sum_{\lambda \in X_{N}} q^{-2(\lambda+\rho, \rho)} Q(\lambda) / Q(0)
$$




$$
\begin{aligned}
& =z^{-1} \Omega(-1)^{\left|\Phi^{+}\right|} N^{r} / Q(0) \\
& =\frac{(-1)^{\left|\Phi_{+}\right|} q^{-\left(1+\frac{N+1}{2}\right)(2 \rho, \rho)}\left(G_{1}(q)\right)^{r}}{Q(0)} .
\end{aligned}
$$

The far right-hand side coincides with $d_{0}^{-1}$. The fact that $\mathscr{F}\left(S^{2} \times S^{1}\right)=d_{0}^{-1}$ can actually be deduced from general arguments, but here we have arrived at this result through direct computations.

\section{Some Remarks}

Recall that the Reshetikhin-Turaev construction is usually formulated in terms of modular Hopf algebras. A modular Hopf algebra is a quasi-triangular Hopf algebra with a distinguished set of irreducible representations which satisfy some rather rigid conditions. The essence of these conditions is recapitulated by Lemma 1, though it should be observed that Lemma 1 does not suffice to make $U_{q}^{(N)}(g)$ qualify as a modular Hopf algebra.

From any given modular Hopf algebra, it is possible to construct a threedimensional topological field theory (see Chapter IV of [6]; for a somewhat different construction, see [32]) of the kind defined by Atiyah [33], by extending the Reshetikhin-Turaev invariant to an invariant of 3-cobordisms. Given Lemma 1, the method of [6] should also apply to the $U_{q}^{(N)}(g)$ with some modifications, though we have not yet gone through the details.

Acknowledgements We thank Dr QH Yin for assistance This work is supported by the Australian Research Council

\section{References}

1 Jones, V F R : Bull Am Math Soc 12, 103 (1985)

2 Kauffman, L H : Knots and Physics Singapore: World Scientific, 1991

3 Witten, E: Commun Math Phys 121, 351 (1989)

4 Kohno, T: Topology 31, 203 (1992); Crane, L: Commun Math Phys 135, 615 (1991)

5 Yu Reshetikhin, N and Turaev, V G : Invent Math 10, 547 (1991)

6 Turaev, V G.: Quantum Invariants of Knots and 3-Manifolds Berlin-New York: Walter de Gruyter, 1994

7 Drinfeld, V G : Quantum groups Proc ICM, Berkeley, (1986), p 798

8. Jimbo, M : Lett. Math Phys. 10, 63 (1985)

9 Turaev, V and Wenzl, H: Inter J Math 4, 323 (1993)

10 Kac, V G : Infinite dimensional Lie algebras 3rd edition, Melbourne: Cambridge Press, 1990, pp $163-167$

11 Lickorish, W B R : Ann. Math 76, 531 (1962)

12 Kirby, R : Invent Math 45, 35 (1978)

13 Fenn, R and Rourke, C: Topology 18, 1 (1979)

14 Axelrod, S and Singer, I : Chern-Simons perturbation theory Proceedings of XXth Conference on Differential Geometrical Methods in Physics, Singapore: World Scientific, 1991, pp 3-45

15 Freed, D and Gompf, R : Commun Math Phys 141, 79 (1991)

16 Jeffrey, L: Commun Math Phys 147, 563 (1992) 
